\title{
Six weeks of $\beta$-alanine supplementation did not enhance repeated-sprint ability or technical performances in young elite basketball players
}

Nutrition and Health 2017, Vol. 23(2) III-118 (C) The Author(s) 2017 Reprints and permission: sagepub.co.uk/journalsPermissions.nav DOI: $10.1177 / 0260106017700436$ journals.sagepub.com/home/nah

\author{
Fabio Milioni, Paulo E Redkva, Fabio A Barbieri and Alessandro M Zagatto
}

\begin{abstract}
Supplementation with $\beta$-alanine plays an important role as a precursor of carnosine, the most effective intramuscular buffer, and has been seen as a potential ergogenic aid, especially for high-intensity modalities such as basketball. Thus, the aim of the present study was to investigate the effects of 6 weeks of $\beta$-alanine supplementation on repeated sprint ability (RSA) and technical performances in young elite Brazilian basketball players. In total, 27 young basketball players (17 \pm I years) were randomized into a $\beta$-alanine group $\left(\mathrm{G} \beta-6.4 \mathrm{~g}\right.$ day $^{-1}$ of $\beta$-alanine) and a placebo group (GP $-6.4 \mathrm{~g} \mathrm{day}^{-1}$ of dextrose). Before and after the supplementation period the athletes performed a RSA test composed of ten $30 \mathrm{~m}$ sprints with two $180^{\circ}$ changes of direction interspaced by $30 \mathrm{~s}$ of recovery. During the recovery period (i.e., after the sprints) the athletes performed a countermovement jump (CMJ) and a set of three free throws. After $48 \mathrm{~h}$ they performed a Yo-Yo intermittent recovery test level I (Yo-YoIRI). Both groups increased the distance covered in the Yo-Yo IRI after the supplementation period $(p=0.00 \mathrm{I})$. On the other hand, both groups presented impairment in RSA time-performance (total time, best time, and mean time, $p \leq 0.04$ ), while no significant changes were observed for technical task performances (i.e., CMJ and free throws) ( $p \geq 0.07)$. No between-group interactions were observed for any variable measured $(p \geq 0.31)$. Thus, 6 weeks of $\beta$-alanine supplementation did not improve RSA or technical performances in young elite basketball players.
\end{abstract}

\section{Keywords}

Repeated sprint ability, Yo-Yo IRI, free throws, jump ability, exercise testing

\section{Introduction}

The ability to perform sprints repeatedly with short recovery periods (Castagna et al., 2007; Padulo et al., 2015b) has been shown as a performance-determinant during basketball games (Padulo et al., 2015b, 2016). Repeated sprint ability (RSA) events are energetically costly and require high demand from both oxidative and non-mitochondrial sources (Gaitanos et al., 1993), which are also called aerobic and anaerobic pathways (for further details on terminology, see Chamari and Padulo, 2015). However, the high utilization of non-mitochondrial energetic sources during high-intensity exercise could cause the accumulation of metabolites, especially $\mathrm{H}^{+}$ions, which can contribute to a decrease in muscle $\mathrm{pH}$ and performance impairment (Bishop et al., 2004; Gaitanos et al., 1993).

The acidosis induced by RSA could also influence the performance of technical tasks (Lyons et al., 2006) such as free throws (Padulo et al., 2015a) and jumps (Carpentier et al., 2015); nevertheless, this issue is poorly addressed in the literature. Thus, strategies to counterattack muscle acidosis could contribute to performance maintenance in both RSA and technical tasks. The amino acid $\beta$-alanine, a combination of $\beta$-alanyl and L-histidine, has been widely investigated as it is a precursor of carnosine, a dipeptide found in high concentrations in skeletal muscle ( $\sim 20 \mathrm{mmol} \mathrm{kg}^{-1}$ in dry weight; Harris et al., 2006), and identified as the most important intramuscular buffer due to its $\mathrm{p} K_{\mathrm{a}}$ value of $\sim 6.8$, within the physiological $\mathrm{pH}$ range 7.1-6.5, acting as the first line of defense against harmful acidosis effects (Abe, 2000).

Department of Physical Education, São Paulo State University (Unesp), Bauru/SP, Brazil

\section{Corresponding author:}

Alessandro M Zagatto, Department of Physical Education, School of Sciences, São Paulo State University (Unesp), Av. Luiz Edmundo Carrijo Coube, I4-0I, Vargem Limpa, CEP I7033-360 - Bauru/SP, Brazil.

Email: azagatto@yahoo.com.br 
Evidence surrounding the ergogenic effects of $\beta$-alanine supplementation is increasing (De Salles Painelli et al., 2014; Hobson et al., 2012; Van Thienen et al., 2009), and Hobson et al. (2012), in a meta-analysis study, demonstrated a performance increase of $\sim 2.85 \%$ when supplementing with at least $179 \mathrm{~g}$ of $\beta$-alanine. These results have also been observed in trained athletes ( Brisola et al., 2016a, 2016b; De Andrade Kratz et al., 2016; De Salles Painelli et al., 2013; Tobias et al., 2013; Van Thienen et al., 2009). Furthermore, chronic supplementation with $\beta$-alanine was effective in reducing the fatigue index when compared to a placebo group during a sequence of 45 jumps (Carpentier et al., 2015), maximizing the shooting skills of elite soldiers with an assault rifle after performing a fatiguing running protocol (Hoffman et al., 2014), and increasing the velocity of shooting in young water polo players (Claus et al., 2017).

Based on basketball game characteristics that involve several RSA actions, as well as the likely potential of $\beta$-alanine as a muscular buffer and the consequent delay in acidosis-induced fatigue, we hypothesized that $\beta$-alanine supplementation would attenuate the performance decrement during repeated high-intensity actions and technical task performance in elite young basketball players.

Therefore, the aim of this investigation was to examine whether $\beta$-alanine supplementation for 6 weeks is able to improve RSA and technical task performances (countermovement jumps and free throws) in young elite Brazilian basketball players.

\section{Methods}

\section{Participants}

Twenty-seven young elite Brazilian male basketball players, in the post-puberty phase, aged 16-19 years (body mass $81.7 \pm 12.6 \mathrm{~kg}$ and height $190.4 \pm 9.7 \mathrm{~cm}$ ), with a minimum of 3 years of competitive experience and approximately $12 \mathrm{~h}$ of training per week were invited to participate in the present study. The inclusion criteria for the study were: (i) participating regularly in previous national competitive seasons; (ii) free from chronic diseases and/or musculoskeletal injuries; and (iii) not having used creatine or protein supplements in the previous 3 months. Participants were instructed to abstain from alcohol or caffeinated beverages during the experimental period and eat a light meal at least $2 \mathrm{~h}$ prior to testing, to reduce interferences in the experiments. During the study there were no athlete transfers or injuries, the only reason for exclusion was noncompliance with the supplementation program (at least $95 \%$ of the total capsules were required to be ingested) or training schedule.

The athletes only participated after being fully informed of the effects, benefits, and potential risks of the study and signing a consent form (or parents/guardians when necessary). All experimental procedures were previously approved by the local Research Ethics Committee (protocol

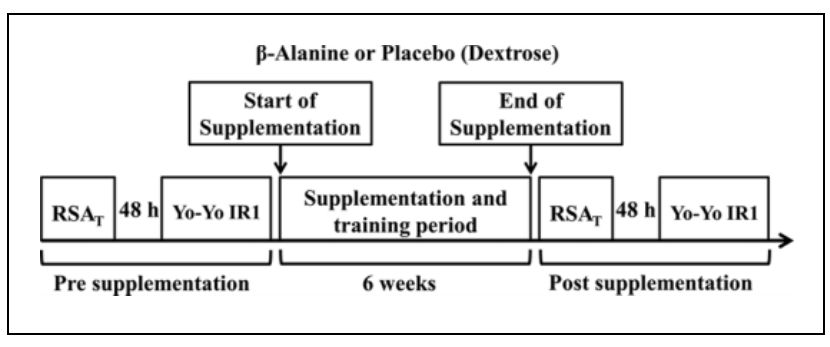

Figure I. Experimental design of the study.

number 1.021.143) according to the ethical principles established by the Declaration of Helsinki.

\section{Experimental design}

Participants were evaluated before and after 6 weeks of supplementation ( $6.4 \mathrm{~g} \mathrm{day}^{-1}$ of $\beta$-alanine or placebo) and training. The protocol test was performed on two different days. All tests were performed in the same period of the day (16:00 to 20:00 - regular training schedule) to eliminate any influence of the circadian cycle and were conducted on a wooden indoor basketball court. Initially, the athletes performed an RSA test with technical tasks $\left(\mathrm{RSA}_{\mathrm{T}}\right.$; Padulo et al., 2015b) and $48 \mathrm{~h}$ later they performed the Yo-Yo intermittent recovery test 1 (Yo-Yo IR1; Castagna et al., 2008). The athletes were divided randomly into two groups; $\beta$-alanine and placebo supplementation groups. A schematic design of the study procedures is shown in Figure 1. During the supplementation period, the training load was monitored in all training sessions using the rate of perceived exertion scale (RPE) CR-10 (Impellizzeri et al., 2004). The athletes were already familiar with testing procedures (i.e., RSA $\mathrm{T}_{\mathrm{T}}$, Yo-Yo IR1, and RPE CR-10) since they formed part of their usual fitness assessment program.

\section{Sample allocation}

The athletes were randomly allocated into two groups, $\beta$-alanine (G $\beta$ ) and placebo (GP - dextrose), only after the first evaluation, considering their playing position (i.e., guards, forwards, and centers) and $\mathrm{RSA}_{\mathrm{T}}$ performance (i.e., higher performance: best sprint time-performance; lower performance: worst sprint time-performance) (Figure 2).

\section{Repeated sprint ability with technical tasks $\left(R S A_{T}\right)$}

To evaluate the influence of RSA on specific basketball technical tasks (i.e., jump capacity and free throw efficacy), the test proposed by Padulo et al. (2015b) was adapted. The test consisted of performing ten 30-m maximal sprints with two $180^{\circ}$ changes of direction $(10 \mathrm{~m}+10 \mathrm{~m}+10 \mathrm{~m})$ interspaced by $30 \mathrm{~s}$ of rest between the sprints (test-retest intraclass correlation (ICC) $>0.90$; Padulo et al., 2015b).

Immediately after each sprint, during the $30 \mathrm{~s}$ rest, the athletes performed a technical task sequence composed of a countermovement jump (CMJ) on a jump platform (Jump Test, Cefise, Nova Odessa, SP, Brazil) and a set of three 


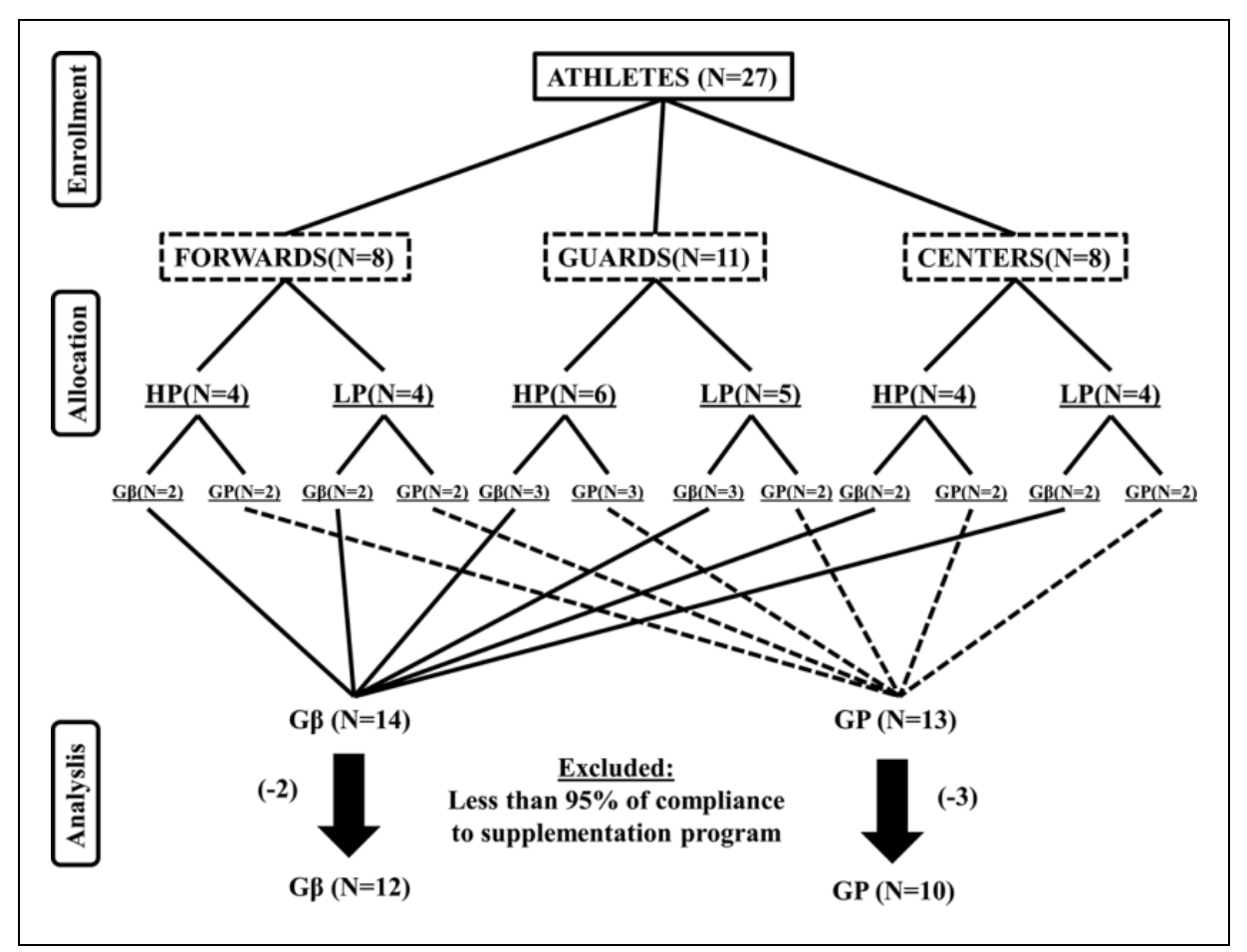

Figure 2. Organizational chart of the sample allocation.

HP: higher performance in RSA ; $_{T}$ LP: lower performance in $\mathrm{RSA}_{T}$; G $\beta$ : $\beta$-alanine group; GP: placebo group.

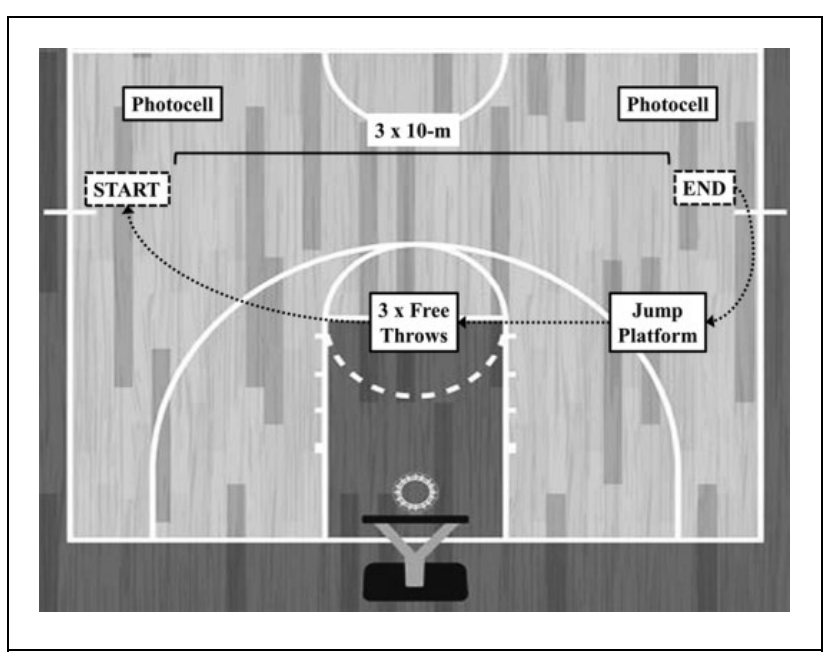

Figure 3. Repeated sprint ability test with technical tasks $\left(\mathrm{RSA}_{\mathrm{T}}\right)$. Dotted arrow is the displacement during $30 \mathrm{~s}$ of rest.

free throw shoots without an interval. After the technical task sequence, which took around $\sim 10 \mathrm{~s}$, the athletes were again positioned at the start line for the subsequent sprint and technical task sequence (Figure 3).

Time of each sprint was recorded by photoelectric cells (Speed Test, Cefise, Nova Odessa, SP, Brazil) positioned at the beginning and end of the track. Therefore, based on the time assessment, the total time (TT - sum of sprint times), best time (BT - best sprint time), worst time (WT - worst sprint time), mean time (MT - mean of sprint times), and percentage decrement were also estimated according to Fitzsimons et al.
(1993) $[\%$ DecT $=100 \times(\mathrm{TT} /(\mathrm{BT} \times 10))-100)]$. Regarding technical tasks, the best jump (BJ - best jump height), worst jump (WJ - worst jump height), mean jump (MJ - mean of jump heights), and percentage decrement $[\% \operatorname{Dec} J=(B J-W J) \times 100) / B J)]$ were measured. The free throw efficacy was also assessed through the total of correct free throw shoots (FT).

\section{Yo-Yo intermittent recovery test level I (Yo-Yo IRI)}

The Yo-Yo IR1 (Castagna et al., 2008) consisted of $40 \mathrm{~m}$ running with $180^{\circ}$ changes of directions $(20 \mathrm{~m}+20 \mathrm{~m})$ with progressive incremental speeds controlled by an audio and $10 \mathrm{~s}$ of active recovery between each $40 \mathrm{~m}$ run, consisting of $10 \mathrm{~m}$ walking $(5 \mathrm{~m}+5 \mathrm{~m})$ (Vernillo et al., 2012). When the participant failed twice consecutively to touch the finish line, exhaustion was assumed. The test score was considered as the total distance covered, including the incomplete final run. For Under 14, 15, and 17 elite Italian young basketball players, the test-retest ICCs were between 0.70 and 0.88 (Bangsbo et al., 2008).

\section{Blood lactate concentration determination}

Blood samples were collected 5 and 7 minutes after the YoYo IR1 and RSA $\mathrm{T}_{\mathrm{T}}$ to determine the peak values of blood lactate concentration. Blood samples $(25 \mu \mathrm{L})$ were collected from the earlobe using heparinized capillary tubes and immediately transferred to Eppendorf tubes containing $50 \mu \mathrm{L}$ of $1 \%$ sodium fluoride for subsequent analysis in an electrochemical lactate analyzer (YSI 2300 STAT, Yellow 
Spring Instruments, Yellow Spring, OH, USA) (measurement error of $\pm 2 \%$ ).

\section{Supplementation strategies}

For the supplementation, $6.4 \mathrm{~g}$ day $^{-1}$ was administered to both groups (i.e., $\beta$-alanine and dextrose) in $800 \mathrm{mg}$ capsules with meals (two capsules per meal - breakfast, lunch, dinner, and with a light meal before sleep), to prevent an overdose generating paresthesia, a momentary alteration in skin sensation. This supplementation dosage is in line with the findings of Hobson et al. (2012) who verified the significant effects of $\beta$-alanine supplementation on exercise performance from the accumulation of $179 \mathrm{~g}$ of $\beta$-alanine. Both supplements, $\beta$-alanine $(99.9 \%$ pure $\beta$-alanine; CarnoSyn, NAI, USA) and the placebo (dextrose - Dextrin, Neonutri, Poços de Caldas, MG, Brazil) were administered in gastro-resistant capsules coated with hydroxypropylmethylcellulose (DrCaps, Capsugel, France). Through individual inquiries, 10 athletes ( 3 from the GP and 7 from the $G \beta$ ) reported isolated occurrences of mild paresthesia. None of the participants were vegetarian and they were instructed to abstain from any nutritional aids or supplements during the study and maintain their regular food behavior. The athletes' meals are controlled by the nutritional coach staff and were the same for all athletes. Before the start and during the period of supplementation, participants were instructed not to comment on any side-effects to their teammates.

\section{Monitoring of training load}

All training sessions were monitored for the duration (minutes) and RPE (Impellizzeri et al., 2004). The 6 weeks of training and supplementation were carried out after 4 weeks of general preparatory phase training, during the specific training phase, and beginning of the competitive period, i.e., during the classification phase of the São Paulo State Championship, without a taper phase. All athletes evaluated were engaged in the same training schedule. The prescription of training sessions was prepared by the physical training staff and did not suffer any interference from the researchers. The training load quantified in each session was assumed as the product between the training duration (in minutes) and the value indicated in the RPE CR-10 (Impellizzeri et al., 2004). The RPE CR-10 scale was always presented to the players around 30 minutes after the end of each training session.

\section{Statistical analyses}

All data are presented as mean \pm standard deviation (SD) and $95 \%$ confidence interval $(\mathrm{CI})$. Initially the Shapiro Wilk test for data normality was performed. The training load data were compared using the independent $t$-test. Two-way analysis of variance (ANOVA) was applied to compare the outcomes with "groups" (GP vs. G $\beta$ ) and the

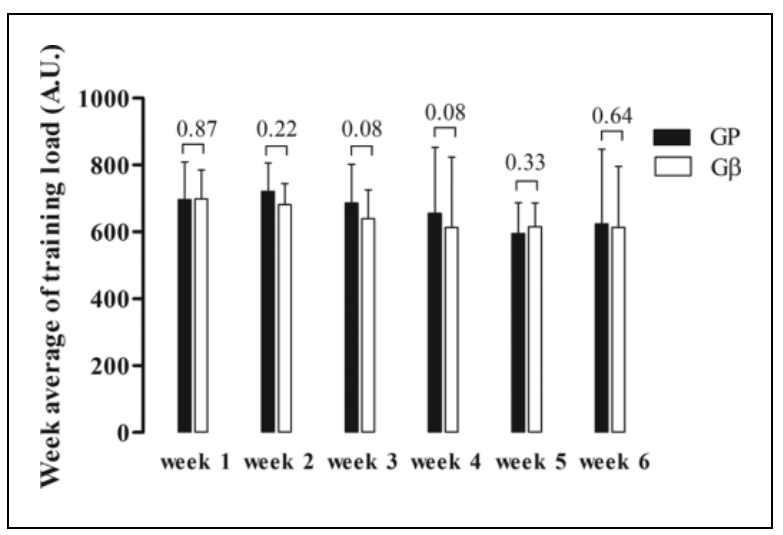

Figure 4. Weekly average of training load. Numbers above the bars represent the $p$-value from an unpaired $t$-test.

interaction between subject factor and "time" (pre vs. post). In addition to ANOVA, the Mauchly sphericity test was applied and sphericity was assumed when no significant $F$ value was observed. In case of violation of sphericity, the epsilon correction of Greenhouse-Geisser was used. The analyses were completed with the Bonferroni post hoc. The effect size (ES) was calculated according to Cohen (1988). The threshold values for Cohen's d statistical power were considered as $>0.2$ (small), $>0.5$ (moderate), and $>0.8$ (large) (Cohen, 1988). Statistical analyses were performed using the software SPSS 21.0. In all cases, a significance level of $5 \%$ was considered.

\section{Results}

The weekly average training load did not differ significantly between groups in each week (Figure 4).

The total distance covered in the Yo-Yo R1 significantly improved from the pre to post supplementation period for both groups $\left(\mathrm{F}_{(1 ; 20)}=14.5, p=0.001\right.$; GP post hoc $p=0.005, \mathrm{ES}=0.34$ and $\mathrm{G} \beta$ post hoc $p=0.04$, $\mathrm{ES}=0.20)$, but no interaction was observed between groups $(\mathrm{GP}$ vs. $\mathrm{G} \beta)\left(\mathrm{F}_{(1 ; 20)}=0.65, p=0.43, \mathrm{ES}=0.03\right)$. The peak of blood lactate after the Yo-Yo IR1 was significantly lower in the post supplementation period only for the $G \beta$ $\left(\mathrm{F}_{(1 ; 20)}=14.5, p=0.001, \mathrm{ES}=0.55 ; \mathrm{GP}-\right.$ pre $\times$ post: post hoc $p=0.06, \mathrm{ES}=0.16$ and $\mathrm{G} \beta-$ pre $\times$ post: post hoc $p=0.001$, $\mathrm{ES}=0.56)$, without interaction between groups $\left(\mathrm{F}_{(1 ; 20)}=4.04, p=0.06, \mathrm{ES}=0.17\right)$ (Figure 5).

Concerning the RSA $\mathrm{T}_{\mathrm{T}}$ outcomes, the statistical analysis showed that the time-performance of the $\mathrm{RSA}_{\mathrm{T}}$ worsened significantly in both groups after 6 weeks (i.e., TT, BT, WT and MT, without significant difference for \%DecT); however, there were no interactions between groups $(1.12>$ $\left.\mathrm{F}_{(1 ; 20)}>0.01,0.96>p>0.31\right)$ (Table 1). The jump capacity, free throw efficacy, and peak of blood lactate concentration were not significantly modified after the $\mathrm{RSA}_{\mathrm{T}}$. In addition, there were no interactions between groups or significant differences in absolute variation (i.e., $\Delta)$ for any of the previous variables $\left(0.81>\mathrm{F}_{(1 ; 20)}>\right.$ $0.03,0.86>p>0.38)($ Table 1$)$. 


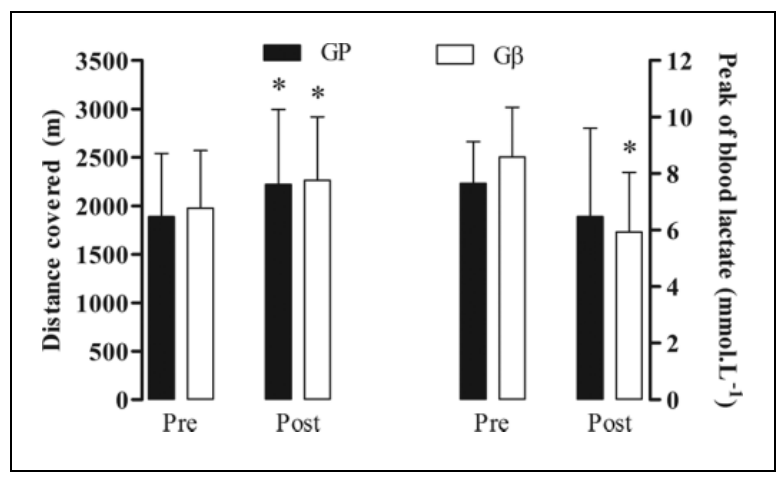

Figure 5. Outcomes from the Yo-Yo IRI. On the left $y$-axis the total distance covered in the Yo-Yo IRI is plotted pre and post supplementation period; on the right $y$-axis the peak of blood lactate is plotted after the Yo-Yo IRI pre and post supplementation period.

*Significant difference from pre supplementation moment withingroup $(p<0.05)$.

\section{Discussion}

The purpose of the present study was to investigate the effects of 6 weeks of $\beta$-alanine supplementation on RSA and technical task performances in young elite Brazilian basketball players. The main finding of the study was the absence of a significant effect of $\beta$-alanine supplementation on performances for both RSA time-performance and technical tasks, since there were no between-group interactions for any variable measured. At the same time a significant improvement in distance covered in the Yo-Yo IR1 was verified for both groups, however this likely occurred as a result of the training.

The Yo-Yo IR1 was developed to evaluate the capacity to perform and recover from intense repeated running efforts (Bangsbo et al., 2008). The protocol is sensitive to assess the aerobic fitness of adult basketball players (Castagna et al., 2008), as well as distinguish athletes with different competitive levels such as elite, sub-elite junior, and non-athletic healthy males (Vernillo et al., 2012). Saunders et al. (2012) found a significant improvement in the distance covered in the Yo-Yo IR2 by amateur soccer players after 12 weeks of $3.2 \mathrm{~g} \mathrm{day}^{-1}$ of $\beta$-alanine supplementation without a difference for the placebo group and a significant interaction between groups.

In the present study, the GP and G $\beta$ presented similar improvement in Yo-Yo IR1 performance. The different outcomes between our study and Saunders et al. (2012) might be due to the Yo-Yo model addressed in each study. While we used the Yo-Yo IR1 that initiates at $8 \mathrm{~km} \mathrm{~h}^{-1}$, Saunders et al. (2012) used the Yo-Yo IR2 that initiates at $11.5 \mathrm{~km} \mathrm{~h}^{-1}$ and likely taxed the glycolytic energy pathway more highly. The high utilization of the glycolytic pathway would have caused an increase in lactate and $\mathrm{H}^{+}$production/concentration, however, the high muscle carnosine content after $\beta$-alanine supplementation, may have buffered the $\mathrm{H}^{+}$action and contributed to increased performance in the Yo-Yo IR2.
On the other hand, only the G $\beta$ presented a decrease in peak of blood lactate concentration after the Yo-Yo IR1. Although muscle carnosine content was not directly measured in the present study, Hill et al. (2007) showed that 4-6 g day $^{-1}$ of $\beta$-alanine supplementation can increase the muscle carnosine content by $40-60 \%$ after 4 weeks of supplementation. The higher muscle carnosine content seems to increase the muscle buffering capacity (Harris et al., 2006) generating a lower $\mathrm{H}^{+}$concentration inside the muscle cells and consequently contributing to the diminished blood lactate value after the Yo-Yo IR1, since the efflux of lactate to the blood stream must be accompanied by $\mathrm{H}^{+}$protons (Bangsbo et al., 1993).

In fact, the significant improvement in distance covered in the Yo-Yo IR1 for both groups must be principally attributed to the systematic training over the 6 weeks, however, in a speculative way, the athletes supplemented with $\beta$-alanine over the training program could have benefited from an increased buffering capacity during this period that would allow higher training intensities/volumes and consequently explain the slightly higher adaptation of the $\mathrm{G} \beta(2264 \pm 653 \mathrm{~m})$ compared to the GP $(2220 \pm 778$ $\mathrm{m})$, despite the difference being non-significant.

Refuting our previous hypothesis, 6 weeks of $\beta$-alanine supplementation did not attenuate the drop in performance during the $\mathrm{RSA}_{\mathrm{T}}$, either for time-performance or technical tasks. The performance decrement presented by both groups in the $\mathrm{RSA}_{\mathrm{T}}$ may be a direct effect of the training phase and competition period, since it is not unusual for athletes to present a drop in performance during the specific training period (Coutts et al., 2007). In addition, no taper phase was allowed before the re-evaluation phase, which would probably result in performance overcompensation after the specific training phase (Mujika and Padilla, 2003).

Recently, Saunders et al. (2016) stated in a metaanalysis study a time frame of $\beta$-alanine action of between 0.5 and $10 \mathrm{~min}$ of exercise duration, showing an overall effect size of 0.18 . This effect size dropped to $\sim 0.11$ when outcomes from exercise protocols that measure performance (i.e., might not elicit maximal effort and are influenced by pace) instead of capacity (i.e., induce volitional exhaustion and generate maximal production of $\mathrm{H}^{+}$) (Saunders et al., 2016) were considered, as in the case of the present study. Furthermore, Hobson et al. (2012) indicated in a meta-analysis that the median effect of $\beta$-alanine supplementation on performance is a $\sim 2.85 \%$ improvement, a range of improvement smaller than the coefficient of variation of the time-performance variables of $\operatorname{RSA}_{\mathrm{T}}(\sim 10.9 \%)$.

Taken together with the $30 \mathrm{~s}$ rest period between sprints, these factors could mask the slight effects of $\beta$-alanine supplementation since the oxidative pathway plays an important role during recovery between high-intensity efforts (Milioni et al., 2017), contributing to phosphocreatine replenishment, even if incomplete, and consequently lower recruitment of the glycolytic pathway (Gaitanos et al., 1993). 


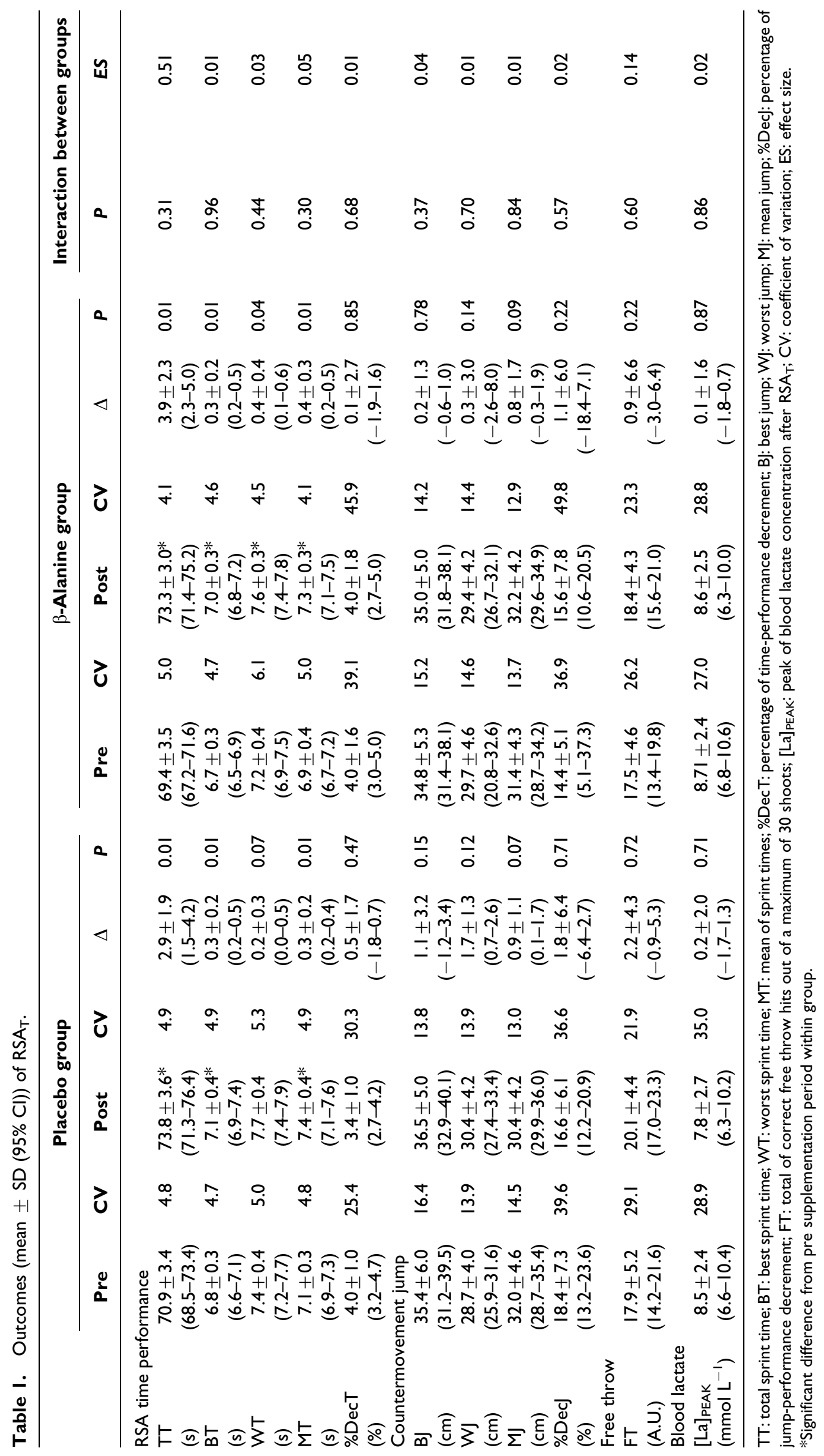


It is important to note that the present study tried to investigate a strategy to improve basketball game performance. In this way, as the test proposed is directly linked to basketball actions (Padulo et al., 2015b), if $\beta$-alanine supplementation could improve $\mathrm{RSA}_{\mathrm{T}}$ performance these results would possibly be transferred to basketball game performance. Corroborating with our findings, Cochran et al. (2015) did not find ergogenic effects of $\beta$-alanine supplementation associated with a training period for RSA measurements.

The inclusion of jumps and free throws after each sprint in the RSA test was an attempt to analyze technical task performances after a high-intensity effort, such as performed during a game. Therefore, the $\beta$-alanine supplementation would act on fatigue over time during a basketball game, which mainly impairs technical task performances such as jumps and free throws.

Ziv and Lidor (2010) reported, in a review paper, high variability of jump measurements in basketball players with an average jump height of between 24.8 and $48.2 \mathrm{~cm}$. Our results of BJ were $\sim 35 \pm 5 \mathrm{~cm}$, corroborating with Ziv and Lidor (2010); however, the CMJ has until now failed to discriminate fatigue in basketball players in conditions such as pre - post games (Castagna et al., 2008; Cortis et al., 2011). The jump, per se, is highly executed during basketball games (i.e., blocking, rebound and shooting; Cortis et al., 2011) and training is performed under maximal and fatigue conditions, in this way it is possible that basketball players develop a high standard of this motor skill as well as specific fatigue resistance that makes them able to perform jumps, even in a fatigued condition, without significant performance decrements (Cortis et al., 2011). Thereby, $\beta$-alanine supplementation may not represent an ergogenic aid for this specific ability in basketball players.

Regarding free throw efficacy, our results are in accordance with previous literature reports, a rate of $\sim 60 \%$ success in shoots performed after exercises at high intensity, such as above $80 \%$ of maximal heart rate (Mokou et al., 2016; Padulo et al., 2015a). Our hypothesis was that $\beta$-alanine supplementation could enhance the workout capacity, delaying the fatigue process induced by RSA and providing conditions to perform the free throws under a better physiological condition, as observed by Hoffman et al. (2014) for elite soldiers during shooting tasks with an assault rifle. However, as for the other performance outcomes, $\beta$-alanine supplementation did not alter the free throw scores.

If on the one hand the absence of significant differences in training loads between groups should be assumed as a positive aspect of the present study, as it isolates the possible outcomes provided by the $\beta$-alanine supplementation, on the other hand, the training stimulus was clearly enough to provide positive adaptations, especially in the Yo-Yo IR1, and this could mask the benefits from $\beta$-alanine supplementation, which in general is around 3\% (Hobson et al., 2012).

Furthermore, despite the $\beta$-alanine supplementation having been proven as efficient to increase muscle carnosine content, with almost 100\% efficacy (Cochran et al., 2015; Hill et al., 2007; Saunders et al., 2017), the main limitation of the present study is the impossibility of a direct measure of muscle carnosine content through muscle biopsy.

Although the present study did not find positive effects of $\beta$-alanine supplementation on young basketball players, Hoffman et al. (2008) verified an increase in volume of resistance training (bench press) as well as decreases in subjective feeling of fatigue after 3 weeks of $4.5 \mathrm{~g} \mathrm{day}^{-1}$ of $\beta$-alanine supplementation. Future research should address the influence of $\beta$-alanine supplementation on training quality during different phases of training periodization and especially on-season, using more sensitive tools such as heart rate variability, hematological and endocrine variables.

Thus, based on these findings, it is possible to conclude that 6 weeks of $\beta$-alanine supplementation did not have any positive effect on the Yo-Yo IR1, RSA outcomes, jumps, or free throw scores performed after high-intensity efforts in young elite basketball players.

\section{Acknowledgements}

Special thanks go to all the participants for their amazing dedication.

\section{Declaration of conflicting interests}

The authors declared no potential conflicts of interest with respect to the research, authorship, and/or publication of this article.

\section{Funding}

The authors disclosed receipt of the following financial support for the research, authorship, and/or publication of this article: by Fundação de Amparo à Pesquisa do Estado de São Paulo (FAPESP; grant numbers 2016/02683-6 and 2016/11076-6).

\section{References}

Abe H (2000) Role of histidine-related compounds as intracellular proton buffering constituents in vertebrate muscle. Biochemistry (Mosc) 65(7): 757-765.

Bangsbo J, Iaia FM and Krustrup P (2008) The Yo-Yo intermittent recovery test: a useful tool for evaluation of physical performance in intermittent sports. Sports Medicine 38(1): 37-51.

Bangsbo J, Johansen L, Graham T, et al. (1993) Lactate and $\mathrm{H}^{+}$ effluxes from human skeletal muscles during intense, dynamic exercise. The Journal of Physiology 462: 115-133.

Bishop D, Edge J, Davis C, et al. (2004) Induced metabolic alkalosis affects muscle metabolism and repeated-sprint ability. Medicine and Science in Sports and Exercise 36(5): 807-813.

Brisola GM, Artioli GG, Papoti M, et al. (2016a) Effects of four weeks of beta-alanine supplementation on repeated sprint ability in water polo players. PLoS One 11(12): e0167968.

Brisola GM, Milioni F, Papoti M, et al. (2016b) Effects of 4 weeks of beta-alanine supplementation on swim performance parameters in water polo players. International Journal of Sports Physiology and Performance. Epub ahead of print 14 December 2016. http://dx.doi.org/10.1123/ijspp.2016-0133.

Carpentier A, Olbrechts N, Vieillevoye S, et al. (2015) Betaalanine supplementation slightly enhances repeated plyometric performance after high-intensity training in humans. Amino Acids 47(7): 1479-1483. 
Castagna C, Impellizzeri FM, Rampinini E, et al. (2008) The YoYo intermittent recovery test in basketball players. Journal of Science and Medicine in Sport 11(2): 202-208.

Castagna C, Manzi V, D'Ottavio S, et al. (2007) Relation between maximal aerobic power and the ability to repeat sprints in young basketball players. Journal of Strength and Conditioning Research 21(4): 1172-1176.

Chamari K and Padulo J (2015) 'Aerobic' and 'Anaerobic' terms used in exercise physiology: a critical terminology reflection. Sports Medicine - Open 1(1): 9.

Claus GM, Redkva PE, Brisola GMP, et al. (2017) $\beta$-Alanine supplementation improves throwing velocities in repeated sprint ability and 200-m swimming performance in young water polo players. Pediatric Exercise Science. Epub ahead of print 25 January 2017. DOI: 10.1123/pes.2016-0176.

Cochran AJR, Percival ME, Thompson S, et al. (2015) $\beta$-Alanine supplementation does not augment the skeletal muscle adaptive response to 6 weeks of sprint interval training. International Journal of Sport Nutrition and Exercise Metabolism 25(6): 541-549.

Cohen J (1988) Statistical Power Analysis for the Behavioral Sciences. Hillsdale, NJ: Lawrence Erlbaum Associates.

Cortis C, Tessitore A, Lupo C, et al. (2011) Inter-limb coordination, strength, jump, and sprint performances following a youth men's basketball game. Journal of Strength and Conditioning Research 25(1): 135-142.

Coutts AJ, Reaburn P, Piva TJ, et al. (2007) Monitoring for overreaching in rugby league players. European Journal of Applied Physiology 99(3): 313-324.

De Andrade Kratz C, de Salles Painelli V, de Andrade Nemezio KM, et al. (2016) Beta-alanine supplementation enhances judo-related performance in highly-trained athletes. Journal of Science and Medicine in Sport. Epub ahead of print 26 August 2016. DOI: 10.1016/j.jsams.2016.08.014.

De Salles Painelli V, Roschel H, Jesus F, et al. (2013) The ergogenic effect of beta-alanine combined with sodium bicarbonate on high-intensity swimming performance. Applied Physiology, Nutrition, and Metabolism 38(5): 525-532.

De Salles Painelli V, Saunders B, Sale C, et al. (2014) Influence of training status on high-intensity intermittent performance in response to beta-alanine supplementation. Amino Acids 46(5): 1207-1215.

Fitzsimons M, Dawson B, Ware D, et al. (1993) Cycling and running tests of repeated sprint ability. Aust J Sci Med Sport 25: $82-87$.

Gaitanos GC, Williams C, Boobis LH, et al. (1993) Human muscle metabolism during intermittent maximal exercise. Journal of Applied Physiology 75(2): 712-719.

Harris RC, Tallon MJ, Dunnett M, et al. (2006) The absorption of orally supplied beta-alanine and its effect on muscle carnosine synthesis in human vastus lateralis. Amino Acids 30(3): 279-289.

Hill CA, Harris RC, Kim HJ, et al. (2007) Influence of betaalanine supplementation on skeletal muscle carnosine concentrations and high intensity cycling capacity. Amino Acids 32(2): 225-233.

Hobson RM, Saunders B, Ball G, et al. (2012) Effects of betaalanine supplementation on exercise performance: a metaanalysis. Amino Acids 43(1): 25-37.

Hoffman JR, Landau G, Stout JR, et al. (2014) Beta-alanine supplementation improves tactical performance but not cognitive function in combat soldiers. Journal of the International Society of Sports Nutrition 11(1): 15.
Hoffman JR, Ratamess NA, Faigenbaum AD, et al. (2008) Shortduration beta-alanine supplementation increases training volume and reduces subjective feelings of fatigue in college football players. Nutrition Research 28(1): 31-35.

Impellizzeri FM, Rampinini E, Coutts AJ, et al. (2004) Use of RPE-based training load in soccer. Medicine and Science in Sports and Exercise 36(6): 1042-1047.

Lyons M, Al-Nakeeb Y and Nevill A (2006) The impact of moderate and high intensity total body fatigue on passing accuracy in expert and novice basketball players. Journal of Sports Science \& Medicine 5(2): 215-227.

Milioni F, Zagatto AM, Barbieri RA, et al. (2017) Energy systems contribution in the running-based anaerobic sprint test. International Journal of Sports Medicine. Epub ahead of print 13 February 2017. http://dx.doi.org/10.1055/s-0042117722 .

Mokou E, Nikolaidis PT, Padulo J, et al. (2016) The acute effect of exercise intensity on free throws in young basketball players. Sport Science for Health 12(2): 227-232.

Mujika I and Padilla S (2003) Scientific bases for precompetition tapering strategies. Medicine and Science in Sports and Exercise 35(7): 1182-1187.

Padulo J, Attene G, Migliaccio GM, et al. (2015a) Metabolic optimisation of the basketball free throw. Journal of Sports Sciences 33(14): 1454-1458.

Padulo J, Bragazzi NL, Nikolaidis PT, et al. (2016) Repeated sprint ability in young basketball players: multi-direction vs. onechange of direction (Part 1). Frontiers in Physiology 7: 133.

Padulo J, Laffaye G, Haddad M, et al. (2015b) Repeated sprint ability in young basketball players: one vs. two changes of direction (Part 1). Journal of Sports Sciences 33(14): 1480-1492.

Saunders B, Elliott-Sale K, Artioli GG, et al. (2016) Beta-alanine supplementation to improve exercise capacity and performance: a systematic review and meta-analysis. British Journal of Sports Medicine. Epub ahead of print 18 October 2016. DOI: 10.1136/bjsports-2016-096396.

Saunders B, Painelli VS, Oliveira LF, et al. (2017) Twenty-four weeks beta-alanine supplementation on carnosine content, related genes, and exercise. Medicine and Science in Sports and Exercise. Epub ahead of print 11 January 2017, DOI: 10. 1249/MSS.0000000000001173.

Saunders B, Sunderland C, Harris RC, et al. (2012) Beta-alanine supplementation improves Yo-Yo intermittent recovery test performance. Journal of the International Society of Sports Nutrition 9(1): 39.

Tobias G, Benatti FB, de Salles Painelli V, et al. (2013) Additive effects of beta-alanine and sodium bicarbonate on upper-body intermittent performance. Amino Acids 45(2): 309-317.

Van Thienen R, Van Proeyen K, Van den Eynde B, et al. (2009) Beta-alanine improves sprint performance in endurance cycling. Medicine and Science in Sports and Exercise 41(4): 898-903.

Vernillo G, Silvestri A and La Torre A (2012) The Yo-Yo intermittent recovery test in junior basketball players according to performance level and age group. Journal of Strength and Conditioning Research 26(9): 2490-2494.

Ziv G and Lidor R. (2010) Vertical jump in female and male basketball players: a review of observational and experimental studies. Journal of Science and Medicine in Sport 13(3): 332-339. 